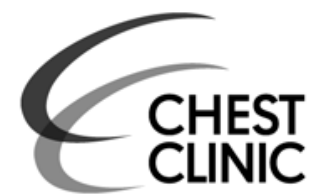

National Pulmonary

Hypertension Service, Adult Congenital Heart Centre and Centre for Pulmonary Hypertension, Royal Brompton \& Harefield NHS Trust, London, UK

\section{Correspondence to}

Dr P Marino, National Pulmonary Hypertension Service, Adult Congenital Heart Centre and Centre for Pulmonary

Hypertension, Royal Brompton \& Harefield NHS Trust, Sydney

Street, London, UK;

p.marino@rbht.nhs.uk

Received 8 June 2011 Accepted 12 December 2011 Published Online First 16 January 2012

\title{
Novel strategies for the management of right ventricular failure with pulmonary hypertension
}

\author{
I C Meryon, P Marino
}

\begin{abstract}
The authors report a case where four novel strategies were successfully used in the management of advanced right ventricular failure with severe pulmonary hypertension and complex adult congenital heart disease. These included combination and optimisation of three advanced therapies for pulmonary hypertension, therapeutic abdominal paracentesis, correction of underlying metabolic/endocrine disturbance and a new inotropic agent.
\end{abstract}

\section{CASE REPORT}

Dr Meryon (ICM): A 36-year-old lady with pulmonary arterial hypertension (PAH) has been admitted with severe right ventricular failure. She has a medical history of double outlet right ventricle with a residual ventricular septal defect following repair in childhood, and associated PAH. Advanced therapies for PAH were commenced 6 years earlier, with an endothelin receptor antagonist (bosentan). Following an initial clinical response, progression of her symptoms and echocardiographic deterioration led to a phosphodiesterase inhibitor (sildenafil) being introduced 2 years later. Further deterioration resulted in the addition of prostanoid therapy (epoprostenol, continuous infusion via a permanently implanted central venous catheter) 6 months prior to admission. Other therapies included oral furosemide, spironolactone and warfarin. Despite these interventions, our patient has been admitted urgently following a short history of abdominal distension, dyspnoea and reduced exercise tolerance.

Dr Marino (PM): This patient has developed advanced right ventricular (RV) failure and $\mathrm{PAH}$ that is apparently refractory to triple combination advanced therapies and conventional medical measures (high dose diuretics, anticoagulation).

The potential therapeutic options include additional treatment to reduce RV afterload and improve preload (abdominal paracentesis) and directly augment RV function (eg, inotropes, correcting any metabolic/endocrine disturbance). We must also ensure that the patient has not developed any other complications commonly associated with her condition such as paroxysmal arrhythmias (seen in end stage disease) or infective endocarditis, which may precipitate acute decompensated heart failure.

All these strategies must be considered in order to optimise her therapy and allow consideration for possible transplant referral.
ICM: On admission, oxygen saturation was $84 \%$ in room air. She was breathless at rest with overt abdominal distension. Blood pressure was 100/ $62 \mathrm{~mm} \mathrm{Hg}$, and radial pulse regular at 76 beats per minute. A systolic murmur was audible at the left sternal edge with a loud P2. The jugular venous pressure was elevated, at the angle of the mandible. There was no peripheral oedema. Chest auscultation was unremarkable. Abdominal examination revealed tense ascites with shifting dullness.

PM: The development of marked ascites poses a serious concern with respect to precipitating further RV compromise and end-organ dysfunction. This may occur through several mechanisms. Ascites raises intra-abdominal pressure (IAP) causing compression of the inferior vena cava, reducing venous return and preload to the RV and compromising systolic function. In addition, raised IAP increases left ventricular afterload thereby diminishing cardiac output to the major organs, while simultaneously increasing venous pressure and congestion causing reduced blood flow and further organ dysfunction.

Early and aggressive treatment of such significant ascites is essential to prevent further cardiac compromise and end organ dysfunction, as well as allowing other measures (eg, inotropy and intravenous diuretics) to treat the underlying cause.

ICM: Admission blood tests revealed a normal haematology, appropriate anticoagulation and mild iron deficiency (transferrin saturation 19\%, Iron $10.8 \mu \mathrm{mol} / \mathrm{l}$ ) Renal function was mildly impaired (GFR $64 \mathrm{ml} / \mathrm{min} / 1.73 \mathrm{~m}^{2}$ ) and electrolytes were within normal ranges. Thyroid-stimulating hormone was raised at $12.40 \mathrm{IU} / 1$ with a normal thyroxine (T4) level.

ECG was sinus rhythm with right bundle branch block, normal axis. Chest radiograph is shown (figure 1). An abdominal ultrasound confirmed ascites and hepatomegaly but no biliary dilatation or focal hepatic pathology. The portal and hepatic veins were prominent with normal flow. Echocardiogram showed severe right ventricular dilatation with marked impairment and compression of the left ventricle but preserved systolic function (figure 2).

PM: I would commence a continuous intravenous furosemide infusion $(10 \mathrm{mg} / \mathrm{h})$ with close monitoring of renal function and urine output. Increasing the spironolactone dose would help to prevent secondary hyperaldosteronism and further ascites. Inotrope therapy, such as dobutamine, could also be considered to augment cardiac output and end organ perfusion, thereby aiding diuresis. 


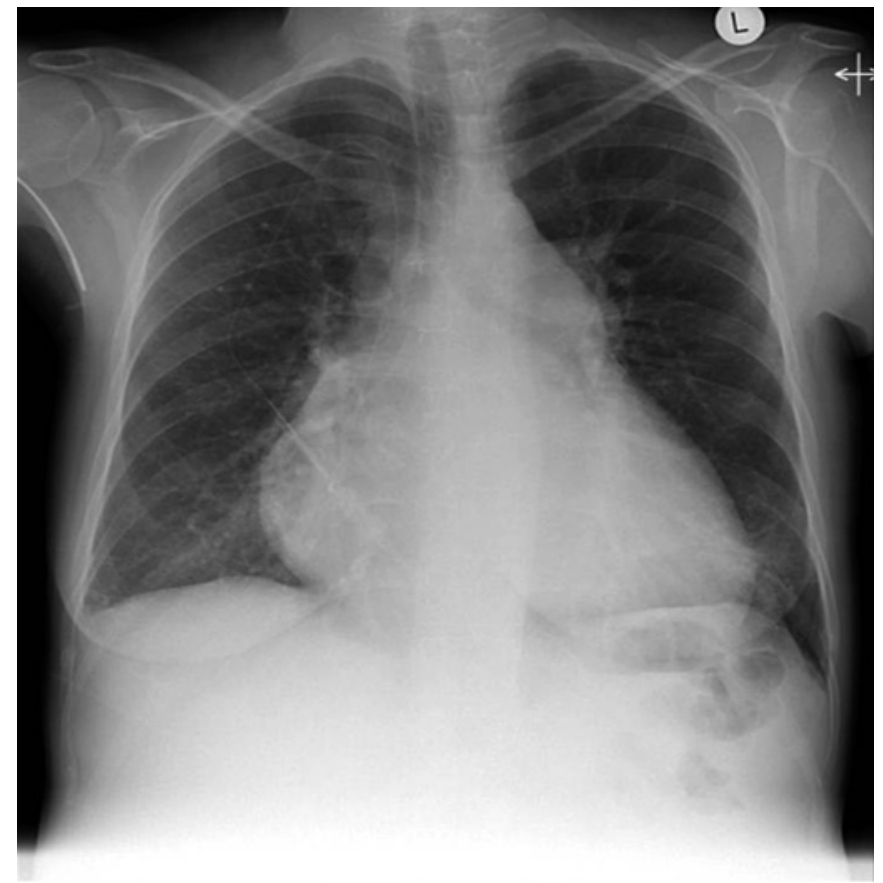

Figure 1 Chest radiograph on admission to hospital.

Hypothyroidism is well recognised in the context of PAH and would introduce low dose levothyroxine to further improve cardiac function in the presence of subclinical hypothyroidism. In addition, these patients are prone to develop significant iron deficiency which can also result in RV dysfunction making iron supplementation essential. ${ }^{1}$

ICM: Despite these measures, her condition has not improved after six days of conventional medical management. Our patient has developed respiratory distress from diaphragmatic splinting by the tense ascites. She developed worsening hyponatraemia and oliguria despite increasing diuretic therapy.

PM: Following non-response to conventional combination diuretic therapy, a two strand approach was taken, involving

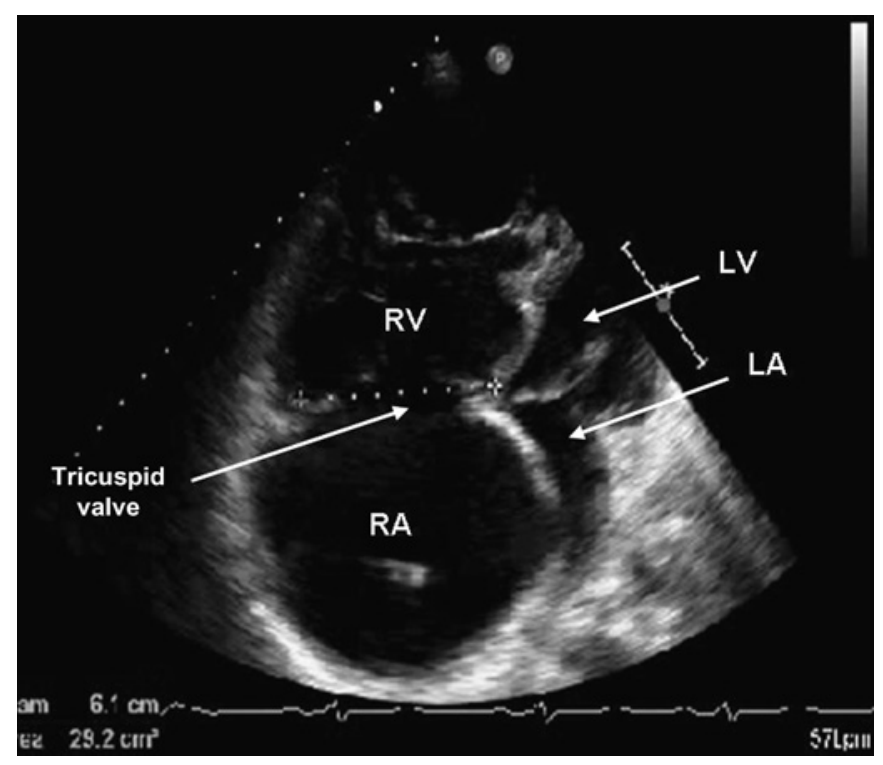

Figure 2 Two-dimensional echocardiogram four-chamber apical view showing severe right ventricular (RV) dilatation with compression of left ventricle (LV). optimisation of pulmonary hypertension and augmentation of right ventricular function. First, the pulmonary hypertension was targeted using triple combination advanced therapies aggressively to reduce RV afterload and aid ventricular function. Therefore, we continued to up-titrate the epoprostenol dose, as tolerated by the patient. Although these agents have a vasodilator effect, their main mode of action is vascular remodelling and therefore an effective clinical response can take several weeks. $^{2}$

The second line of approach was to support the right ventricle until triple therapy optimisation was established. ${ }^{3}{ }^{4}$ Two key strategies were used in this case.

Additional inotropic support was introduced using levosimendan, which has three potential physiological benefits; this new agent is a potent pulmonary vasodilator, while providing a strong inotropic effect on the right ventricle and augmenting diastolic function. These effects are attractive as these are achieved without significantly increasing myocardial oxygen demand. However, there are two potential adverse effects: profound systemic hypotension (potentiating the side effects of the advanced therapies) and lowering the threshold for atrial tachyarrhythmias. Therefore, its use has been limited to the critical care setting. ${ }^{5}$

In addition, controlled therapeutic paracentesis is an attractive intervention in advanced right ventricular failure. This increases renal blood flow through raised cardiac output, allowing afferent arteriolar pressure to rise in the glomerulus. Furthermore, a reduction in IAP will augment venous return by reducing inferior vena cava compression, thereby improving right ventricular filling. However, paracentesis can potentially cause catastrophic large volume fluid shifts, so the procedure was undertaken under controlled circumstances on the high dependency unit, with simultaneous replacement using saltdepleted human albumin solution. ${ }^{6}$

Several factors increased the risk of intra-abdominal bleeding with this invasive procedure (where haemostasis would be difficult). Anticoagulants and hepatic congestion resulted in a prolonged prothrombin time which proved refractory to prothrombin complex concentrate. Vasodilator therapies potentially impair haemostasis through prevention of protective vasospasm, while prostacyclin's antiplatelet action further increased the bleeding risk of this intervention.

Finally, sepsis remains a concern in a patient with a central venous catheter and abdominal drains. Therefore, the abdominal drain was removed immediately after each paracentesis, and prophylactic antibiotics were administered.

ICM: A therapeutic abdominal paracentesis was performed, draining 31 of ascitic fluid with concomitant salt poor human albumin solution replacement. The dobutamine was weaned and levosimendan was commenced, in combination with a second therapeutic paracentesis procedure. All remaining ascitic fluid was removed (5.5 l) with appropriate salt poor human albumin replacement.

This has resulted in significant clinical improvement. There was no re-accumulation of ascites, renal function stabilised with a sustained diuresis. Ultimately, these interventions meant inotropes could be fully weaned with sustained clinical response and be discharged home. This optimised her sufficiently to facilitate assessment for transplantation.

PM: This case highlights:

1. The use of combination triple advanced pulmonary hypertension therapies at maximal dose.

2. The importance of treating co-existent metabolic and endocrine disorders that commonly arise in $\mathrm{PAH}$ patients. 
3. The role of a new inotrope, levosimendan in advanced RV failure.

4. A novel use of controlled therapeutic abdominal paracentesis in right ventricular failure refractory to conventional medical management.

Competing interests None.

Provenance and peer review Not commissioned; externally peer reviewed.

\section{REFERENCES}

1. Tay EL, Peset A, Papaphylactou M, et al. Replacement therapy for iron deficiency improves exercise capacity and quality of life in patients with cyanotic congenital heart disease and/or the Eisenmenger syndrome. Int J Cardiol 2011;151:307-12.
2. Archer SL, Michelakis ED. An evidence-based approach to the management of pulmonary arterial hypertension. Curr Opin Cardiol 2006;21:

$$
\text { 385-92. }
$$

3. Zamanian RT, Haddad F, Doyle RL, et al. Management strategies for patients with pulmonary hypertension in the intensive care unit. Crit Care Med 2007:35:2037-50.

4. Price LC, Wort SJ, Finney SJ, et al. Pulmonary vascular and right ventricular dysfunction in adult critical care: current and emerging options for management: a systematic literature review. Crit Care 2010;14:R169.

5. Follath F, Cleland JG, Just $\mathrm{H}$, et al. Efficacy and safety of intravenous levosimendan compared with dobutamine in severe low-output heart failure (the LIDO study): a randomised double-blind trial. Lancet 2002;360:196-202.

6. Mullens W, Abrahams Z, Francis GS, et al. Prompt reduction in intra-abdominal pressure following large-volume mechanical fluid removal improves renal insufficiency in refractory decompensated heart failure. J Card Fail 2008;14: 508-14. 